\title{
IMPORTANCE OF TEXTURE DESCRIPTORS IN PRODUCT MANUFACTURING INSPECTION
}

\author{
SIR-ALEXCI SUAREZ ${ }^{1}$, ISBELIA KARINA RINCON ${ }^{2} \&$ ALBERT MIYER SUAREZ $^{3}$ \\ 1, 2 Engineering Faculty, University Francisco of Paula Santander Ocaña, Colombia \\ ${ }^{3}$ Faculty of Engineering and Architecture. University of Pamplona, Colombia
}

\begin{abstract}
This article presents an analysis of the use of texture descriptors extracted from images in the manufacture of products, through inspection with visual systems that incorporate such descriptors. $A$ wide description is made in the industry and the importance in the inspection of pharmaceutical, textile, food and metal surfaces is determined. It also describes the classification methods and the configuration of the environment in the data extraction process, which may include noise if the appropriate lighting characteristics are not taken into account, which results in the limitation of the recognition systems. It also highlights the importance for industries to reduce delivery time, decrease manufacturing costs and defects found in each product.
\end{abstract}

KEYWORDS: Inspection of materials, artificial vision, texture descriptors, product manufacturing

Received: Nov 28, 2020; Accepted: Dec 18, 2020; Published: Dec 26, 2020; Paper Id.: IJMPERDDEC202063

\section{INTRODUCTION}

The inspection that is carried out in the manufacture of products is a task that can reduce the specific errors given by means of a list of tasks that allows to know when a product is within the percentage of being accepted under quality standards. The process can be performed by trained personnel who perform tasks by time cycles, and can be molded if the specifications change, in general can work when it comes to the manufacture of few units, but if production is high surely the products can reach the final customer with serious problems and manufacturing errors. According to the above, artificial vision systems are still implemented to reduce the time in finding defects in each product and help to decrease the delivery time and improve the safety level where each manufactured product will be used.

The vision systems use descriptors that are extracted by the characteristics of the images taken on the assembly line, and can detect errors ranging from defects to noise and product contamination.

Inspection systems must be reconfigured according to the product, and work to improve their usefulness is changing daily, implementing new visual techniques due to the great variety of defects and changing products that the market demands. The goal is always to improve the success rate, decrease the processing time and reduce the cost of inspection systems that use artificial vision. The importance of texture descriptors used for inspection in different areas such as metals, medicine, glass, textiles and food is determined.

\section{METHODOLOGY}

A process is carried out where the most important industries are analyzed, and subsequently from each industry the techniques and methods used are analyzed according to the product to be examined, a correlation is made of the 
common techniques that serve for different products in each of the industries. Once the technique used is detected, the best configuration is shown according to the texture descriptors and the types of material and lighting that must be followed for acceptable recognition.

The vision system devices and the importance of their use and cost are presented, to finally make a discussion of the processes that are being implemented in each of the industries with the greatest potential worldwide. The importance of the texture extracted from the images and the statistical descriptors that can be calculated in each image are presented.

\section{RESULTS AND DISCUSSIONS}

To improve manufacturing control, adequate techniques are needed, such as automatic visual inspection, a quality control process that through image processing techniques such as texture descriptors can determine if the product conforms to the specifications required by the established standard. The ideal objective is to detect all defective products. Normally, pattern recognition is used using three processes: segmentation, in which regions of the images are obtained that correspond to possible defects; feature extraction and classification, in which the segmented regions are separated into two classes: 'defects' and 'regular structures'. The detection is done in the classification, which aims to reduce the number of false positives. The following are the most important results in the industry and a discussion is made based on the importance of each of them.

\subsection{Inspection in Metallic Materials}

Countless methods have been applied using texture for the control of metal parts, such as the detection of defects in aluminum or steel parts. Texture is also used for automatic defect detection in cast parts. One of the problems is finding the type of defects that appear in the casting molds of materials such as iron and steel. The first works generally consisted of using a television camera and a microcomputer. Through the acquisition of an image $(8$ bits) they extract the appropriate characteristics, illuminating the surface and using a smaller window of the image with the aim of diminishing the effects of reflection.

Some of the interesting works are those developed for the automatic detection of defects in aluminum castings through the analysis of digital radioscopic images and texture patterns. They present an exhaustive analysis of more than 400 characteristics extracted in almost 23,000 regions from 50 radioscopic images. The extracted features were grouped into geometrics and intensity (14 Haralick descriptors of the co-occurrence matrix). A receiver operation characteristic (ROC) analysis was presented with which it is possible to measure the reliability of each characteristic individually. The image is obtained by irradiating the part with X-rays. These are then converted to visible light by means of an image amplifier. Through an analog-digital converter, the electrical signal is converted into a binary code that can be interpreted by a computer to form a digital image. The images are classified in two ways: 'defects' and 'regular structures' using statistical classifiers, linear and neural networks. They also analyze images of the surfaces of parts that have undergone machining operations and investigate the correlation between wear and the variables that characterize these surfaces by means of column projection analysis and Run Length statistics.

In the field of polishing and grinding operations, it is important to inspect and classify possible defects on the product surfaces in order to obtain high quality in both functionality and aesthetics.

It can be applied to systems for recognizing the position and orientation of complex workpieces in the task of assembling micro-devices, as well as in the quality of surface inspection systems. The reliability of the sorter proposal was 
tested in a database of different images with different mechanical processes.

Another aspect to take into account is the quality of the material with which the pieces are manufactured. The quality of the steel can be evaluated by measuring the texture properties corresponding to the carbide distribution. Normally the quality of this material is carried out manually under a light microscope, where the size of the carbide agglomerations and the direction of distribution are reflected. They develop an image processing tool based on morphological operations, Gabor filters and scale space methods, to capture these textural properties. In particular, to reliably estimate the dominant size in the image structures, they explored automatic scaling techniques and the use of this information in the calculation of texture descriptors.

Another work developed to inspect the steel in order to detect four types of defects: the color of the steel, patches, holes and marks. It is based on the use of neural networks and the color histogram. One of the drawbacks when extracting the color characteristics is the dependence of the images on several variables, such as the position of the camera and the illumination, so the system needs to be reconfigured in different positions. Holes or holes, which are actually the discontinuities on the surface of the steel, are characterized by dark backgrounds; therefore, an adequate level of illumination is needed for detection. The image is divided into a series of non-overlapping blocks and treated one by one using Gabor Filters to extract the characteristics of the image.

The texture of the machined surfaces provides reliable information on the degree of tool wear. A job where the images are first pre-processed by edge detection using the Canny algorithm, the Hough transform is applied to the image to detect the edge of all line segments, finally the distribution of line segment orientations and lengths are used to determine tool wear. Similarly, they classify surface wear particles and study texture and shape characteristics according to three processes: fatigue, adhesion and abrasion. The characteristics extracted for this study were a combination of the Wavelet Transform and the Cooccurrence Matrix. As a conclusion it is deduced that the wear particles of the abrasion process were the easiest to distinguish; the size of parameters such as area and perimeter can be used to distinguish between fatigue, abrasion and adhesion particles and the texture patterns offer a low percentage of error in the classification.

The defects of undercutting by welding, are analyzed determining the advantages and disadvantages of visual systems against humans, explaining the entire process of implementing an automatic system where all points of interest of welding are analyzed. The defects of metallic materials are also classified by means of SVM, implementing a system based on the supervision of human vision, managing to extract the same patterns that an operator in charge of the inspection of the material achieves [1]. Human inspection also has some advantages, such as the ease with which a human being can adapt quickly to changes in the process [2], a situation in which an automated system requires new tests and a different configuration. The sale of human inspection is found in companies with little production, while if production is high, there is a risk of bringing to the final buyer a product that does not have the technical specifications.

The metals maintain a peculiarity due to visual perception [3-4], given by the illumination where the brightness and contrast can affect the capture and extraction of data, that is why constant changes are made by varying the light or placing the object in cells that isolate the environment to obtain a better view when taking each of the images of the product to be inspected.

\subsection{Inspection of Textile Materials}

The inspection of different materials through texture analysis has been widely studied in various industrial processes such as 
the manufacture of textiles, food, paper, leather, wood, cork, ceramics, medicine, etc. Quality control is one of the main tasks in the textile industry. The works apply the cooccurrence matrix, focusing on the change of illumination, size variation and distance variation, and how each of the descriptors proposed by Haralick is affected when examining different types of textiles.

They also present new inspection algorithms for textile quality. The proposed system differs from existing systems in two ways: low cost and easy assembly. The system is configured from a combination of inspection with binary image processing, texture analysis and neural networks. The inspection algorithm is composed of two modules: the defect detection and feature extraction and the second module the classification. The first module determines the region where the information will be extracted and consequently the defect. The texture characteristics extracted were contrast, second angular momentum, mean, entropy and homogeneity. The vision machines have changed the textile world [5], their increasing incorporation and the low prices to acquire these systems, placing Asia at the forefront of textile production with $68 \%$ globally, and the visual systems allow to deliver to the buyer a product with few defects increasing the profitability for the company that implements those [6].

The applications that are being implemented most in the textile industry are: defect identification, color correlation and pattern management [7], which allows to determine the quality of the textile product using all the power of computers with artificial intelligence incorporated into increasingly robotic devices.

\subsection{Inspection in the Food Industry}

In the food industry, statistical texture descriptors are the most commonly used to detect and classify product quality. Within these methods, the co-occurrence matrix has been applied to discriminate different foods such as French fries and cooked potatoes, poultry meat, identify cereal grains, predict the chemical properties of oranges, analyze changes in the surface of chocolate, classify beef among other food products.

The taste, color and texture of the French fries is important to achieve a product that can compete in the market. One of the works that addresses this problem is done using color pattern recognition to classify French fries.

In the same way, the texture serves to analyze different types of fruits such as apples and oranges. In this field, another research using neuronal networks and GLCM was developed by [8] with the aim of studying the type of orange Iyokan, since it is generally believed that the reddish color of the orange gives a sweet taste to the fruit. Therefore, in this work, the sugar content and acidity of the orange are analyzed. The textural characteristics were the second angular moment, inverse difference moment and contrast. The images were reduced to 64 gray levels with a distance of 16 pixels and an angle of $0^{\circ}$. In summary, the results demonstrate that the textural characteristics show a very low correlation with the roughness obtained manually; also the research found a low correlation between the appearance of the fruit and the sugar [9].

More recently, products such as cheese, pizza and everything related to baking are being studied by classification systems that can even help detect not only their external appearance, but also their taste and texture using neural networks [10-11].

\subsection{Inspection in Medicine and other Materials}

In materials such as glass, ceramics, plastic or construction waste, the different methods of extracting texture characteristics by visual means have also been analyzed. An article published by [12] examines such extraction in ceramic materials, where 
the process is usually carried out by binary operators. In addressing the problem they found that ceramics are categorized by measuring characteristics such as: hardness, porosity and texture etched into the ceramic, which determine whether a tile is defective or not. The materials selected were 60 ceramics, of which 30 were good and 30 were defective. The training was carried out with 10 images of good quality and 10 images of defective ceramics and the rest were used for the test. The texture methods applied are the characteristics obtained from the first order descriptors (mean, variance, skweness, kurtosis and entropy), GLCM with four directions $0^{\circ}, 45^{\circ}, 90^{\circ}$ and $135^{\circ}$ selecting energy, entropy, homogeneity, inertia, correlation, cluster shade and cluster prominence, with distances of 1 and 2. The results show that homogeneity can be used to characterize very well in ceramics.

It is not only necessary to look at the quality of the product, vision systems are being used to detect the contamination that a medicine may have [13]. This monitoring is done through the same checklist used in the product control area by human operators, so that they take it to the visual system [14-15].

\section{CONCLUSIONS}

The importance of machine vision systems in product inspection and the continuous use of texture measurement present in the images has been determined as a method to determine if it complies with the quality protocols of each industry. The works where they are incorporated have been analyzed due to their high demand and high world production such as: medicine, food, textiles and metals. The researches carried out have determined the good functioning using descriptors in the spatial domain, which analyze the neighboring pixels by means of the defects, color selection and characteristic patterns to determine the parameters of each product.

\section{REFERENCES}

1. Zhang P, Cheng T. Research on metallic material defect detection based on bionic sensing of human visual properties. En 2018. p. 030012 .

2. Budd G. Inspection of Machined Metal Surfaces. 2020. https://www.qualitymag.com/articles/90786-inspection-of-machinedmetal-surfaces? $v=$ preview

3. PTJ. An Exploratory Data Analysis Of Pipeline Coating Degradation | Pipeline Technology Journal. 2020. Disponible en: https://www.pipeline-journal.net/articles/exploratory-data-analysis-pipeline-coating-degradation

4. Todd JT, Norman JF. The visual perception of metal. J Vis. 1 de march de 2018; 18(3):9-9.

5. Ahearn G. A New Look: How Machine Vision Is Changing Textile Inspection | Possibility| Teledyne Imaging. 2020. Disponible en: https://possibility.teledyneimaging.com/a-new-look-how-machine-vision-is-changing-textile-inspection/.

6. Bharadwaj R. Artificial Intelligence in the Textile Industry - Current and Future Applications. 2020. Disponible en: https://emerj.com/ai-sector-overviews/artificial-intelligence-in-the-textile-industry-current-and-future-applications/.

7. Thomas-Industry. 3 Ways Textile Manufacturers are Seamlessly Integrating AI into Production. 2019. Disponible en: https://www.thomasnet.com/insights/3-ways-textile-manufacturers-are-seamlessly-integrating-ai-into-production/.

8. Kondo N, Ahmad U, Monta M, Murase H. Machine vision based quality evaluation of Iyokan orange fruit using neural networks. Computers and Electronics in Agriculture. 2000; 29(1-2):135 - 147.

9. Ali SSE, Dildar SA. An Efficient Quality Inspection of Food Products Using Neural Network Classification. J Intell Syst. 9 de mayo de 2019; 29(1):1425-40. 
10. Campos M. Automation and Manufacturing Innovations for the Food Industry [Internet]. Machine Design. 2018. https://www.machinedesign.com/automation-iiot/article/21836677/automation-and-manufacturing-innovations-for-the-foodindustry.

11. Riley S. Food Safety of Packaging Equipment: A Closer Look - Food Safety Magazine [Internet]. 2019. Disponible en: https://www.foodsafetymagazine.com/enewsletter/food-safety-of-packaging-equipment-a-closer-look/.

12. Novak I, Hocenski Z. Texture feature extraction for a visual inspection of ceramic tiles. Industrial Electronics, 2005. ISIE 2005. Proceedings of the IEEE International Symposium on. 2005 June;3: 1279-1283.

13. Wilson A. Vision system detects pharmaceutical contaminants. Vision Systems Design. 2018. https://www.visionsystems.com/factory/consumer-packaged-goods/article/16739310/vision-system-detects-pharmaceutical-contaminants

14. Schiavetti B, Wynendaele E, Melotte V, Van der Elst J, De Spiegeleer B, Ravinetto R. A simplified checklist for the visual inspection of finished pharmaceutical products: a way to empower frontline health workers in the fight against poor-quality medicines. J Pharm Policy Pract. 1 de mayo de 2020; 13(1):9.

15. AIA. Embedded Vision Applications in the Pharmaceutical Industry [Internet]. Vision Online. 2019. https://www.visiononline.org/blog-article.cfm/How-Embedded-Vision-Technology-is-Used-in-the-PharmaceuticalIndustry/148. 